\title{
Anabases
}

ANABASES Traditions et réceptions de l'Antiquité

23 | 2016

Varia

\section{L'Antiquité dans les nouveaux programmes de collège : mise en perspective historique}

Aurélie Rodes

\section{(2) OpenEdition}

1 Journals

Édition électronique

URL : http://journals.openedition.org/anabases/5645

DOI : 10.4000/anabases.5645

ISSN : 2256-9421

Éditeur

E.R.A.S.M.E.

Édition imprimée

Date de publication : 2 mai 2016

Pagination : 185-199

ISSN : 1774-4296

\section{Référence électronique}

Aurélie Rodes, "L'Antiquité dans les nouveaux programmes de collège : mise en perspective

historique », Anabases [En ligne], 23 | 2016, mis en ligne le 02 mai 2019, consulté le 10 décembre 2020

URL : http://journals.openedition.org/anabases/5645 ; DOI : https://doi.org/10.4000/anabases.5645

(c) Anabases 
Anabases 23 (2016), p. I85-i99.

\section{L'Antiquité dans les nouveaux programmes de collège: mise en perspective historique}

Aurélie Rodes

$L^{\prime}$

annonce des nouveaux programmes d'histoire a suscité de multiples réactions, attestant des enjeux que représente encore cette discipline aux yeux tion de nouveaux programmes est accompagnée, depuis le $\mathrm{XIX}^{\mathrm{e}}$ siècle, de critiques récurrentes sur la place des humanités, de l'histoire nationale, de la continuité historique et plus récemment sur le fait religieux et la place des victimes dans l'enseignement. L'élaboration des programmes fut d'abord confiée à des historiens reconnus, tels Ernest Lavisse en i89o, Charles Seignobos en igo2, Lucien Febvre en I945 ou impulsée par Fernand Braudel à la fin des années ig5o. Dans les années I980, du fait de la multiplication des groupes de pression, médiatiques, politiques, universitaires ou encore religieux, les acteurs chargés de l'écriture des programmes se sont diversifiés, s'ouvrant aux représentants syndicaux des enseignants et aux parents d'élèves. Désormais, les propositions sont formulées par le Conseil supérieur des programmes ${ }^{1}$. En avril 20I5, la parution des projets de programmes pour la rentrée 2016 a aussi fait l'objet de réprobations, comme celles dont Pierre Nora ${ }^{2}$ s'est fait le porte-parole. La ministre de l'Éducation nationale a alors invité des historiens autour de tables rondes et demandé une réécriture des programmes d'histoire, en tenant compte des remarques des opposants au changement. Après

1 Les programmes français se présentent sous la forme d'arrêtés ministériels et ne font pas l'objet de votes parlementaires.

2 http://www.lejdd.fr/Societe/Education/L-historien-Pierre-Nora-sur-les-nouveauxprogrammes-d-histoire-L-image-d-une-France-fatiguee-73o638 
une seconde version proposée en septembre, les programmes ont été publiés au bulletin officiel en novembre. Ils ne présentent quasiment aucune modification en ce qui concerne l'Antiquité, ce qui n'est pas surprenant, cette période n'ayant guère été évoquée dans les débats. Cela s'explique, d'une part, parce que l'histoire de l'Antiquité n'est plus perçue comme un enjeu majeur de l'école ${ }^{3}$ et, d'autre part, parce que la question des lettres classiques a focalisé l'attention, pour cette période, sans que le lien n'ait été fait entre l'enseignement de l'histoire et celui des langues anciennes ${ }^{4}$.

Pour comprendre pourquoi les réactions aux nouveaux programmes d'histoire n'ont pas porté sur l'Antiquité, il convient de revenir sur leur évolution depuis la fin du $\mathrm{XIX}^{\mathrm{e}}$ siècle, avant de montrer en quoi les propositions actuelles présentent pourtant des perspectives nouvelles.

\section{L'Antiquité: une part décroissante dans les programmes depuis 1902}

Revenir sur les réformes des programmes d'histoire des $\mathrm{xIX}^{\mathrm{e}}$ et $\mathrm{xx}^{\mathrm{e}}$ siècles, c'est d'abord l'occasion de connaitre la genèse de l'organisation actuelle de l'enseignement de l'histoire dans l'enseignement secondaire. Le dispositif a peu évolué, si ce n'est que, jusqu'à la Seconde Guerre mondiale, l'Antiquité était considérée comme une période majeure de l'histoire, dominant toutes les autres.

$\mathrm{Au} \mathrm{xIx}^{\mathrm{e}}$ siècle, dans l'enseignement secondaire, l'histoire était divisée en trois niveaux: la division élémentaire, réservée à l'enseignement de l'histoire nationale, la division de grammaire consacrée à l'étude de l'histoire ancienne et la division supérieure. L'histoire ancienne était alors répartie sur trois années: l'Orient en classe de sixième, la Grèce en cinquième et l'histoire romaine en quatrième, ce qui conférait à l'Antiquité une place très importante dans l'enseignement de l'histoire. En i88o, Fustel de Coulanges, membre de la commission chargée de préparer un nouveau plan d'étude, proposa de ne pas conserver le continuum chronologique entre les deux cycles: la division grammaire $-6^{\mathrm{e}}, 5^{\mathrm{e}}$ et $4^{\mathrm{e}}$ - et la division supérieure qui correspond au lycée aujourd'hui. Le même programme était alors abordé deux fois de suite, en tenant compte de la maturité des élèves, réservant l'étude de la société et des civilisations pour le premier cycle et les faits plus précis pour le second. Ce dispositif perdure aujourd'hui.

3 A. Ropes, La réception de l'histoire romaine dans l'enseignement secondaire de I880 à nos jours, thèse de doctorat soutenue en 2012, sous la direction de Corinne Bonnet, à l'université Toulouse-Jean Jaurès.

4 Cf. N. MAury, «Latin et grec dans l'enseignement secondaire: une approche historique », dans ce numéro. 
Une autre réforme importante fut adoptée en I902; elle limitait l'histoire ancienne à la seule classe de sixième, au profit de l'histoire contemporaine. Cette réforme constitua une véritable rupture et provoqua de multiples réactions. Les auteurs de manuels s'accommodèrent des réductions de programme, tel Albert Malet, qui avait participé aux commissions pour leur révision en histoire-géographie. Il expliqua, dans les avertissements du manuel qu'il publia chez Hachette, qu'il avait cherché à se conformer au programme, en privilégiant les aspects les plus concrets pour aborder en une seule année les trois civilisations antiques. Les réorganisations dues à cette réforme bouleversèrent profondément les habitudes tant dans les méthodes que dans les contenus et l'instauration de la parité des sciences et des lettres focalisa le débat sur la place des humanités classiques dans les années d'après la Grande Guerre. Durant la guerre, la culture classique fut invoquée pour justifier et exacerber l'opposition franco-allemande. Selon le ministre de l'Éducation Albert Sarraut, l'Antiquité était même au cœur des enjeux du conflit mondial; c'est ce dont il voulait convaincre les élèves lors du discours de la rentrée de igi5.

La culture classique, d'autre part, doit rester l'objet d'une fervente étude, ne serait-ce que parce qu'elle a transmis à la pensée française la plupart des grandes idées pour lesquelles nous combattons. [...] N'est-ce pas d'ailleurs, parce que l'antiquité a toujours été en France, non point seulement un objet de sèche érudition, comme de l'autre côté du Rhin, mais encore un sujet d'admiration et un guide de conduite, que la pensée antique a nourri l'âme française et que le grand conflit actuel est devenu le conflit de deux cultures inconciliables ${ }^{5}$ ?

Les Italiens ayant rallié les Français en mai ıgı5, il était désormais possible de mettre en avant l'héritage antique et d'opposer, comme dans les années i87o, les Latins aux Germains.

Pendant l'entre-deux-guerres, quatre nouveaux programmes d'histoire furent publiés, en réponse aux mécontentements suscités par la réforme de igo2. Ils donnèrent lieu à de nombreux articles et à des débats à la Chambre des députés, notamment en $1922^{6}$. Des hommes politiques et des universitaires prirent alors la défense des humanités classiques:

5 A. Sarraut, “Pour la rentrée d'octobre I9I5 », Revue internationale de l'enseignement, 69 , I9I5, p.32I.

6 Ces débats ont été étudiés par J. BAstier, «La querelle des humanités gréco-latines et la politique de l'éducation de Léon Bérard (I92I-I924)", p.533-574, in M. Ganzin, L'influence de l'Antiquité sur la pensée politique européenne (XVT'-XXe), СERHIP, IX, Presses universitaires d'Aix-Marseille, 1996. 
Si l'on ne veut pas vivre comme un aveugle dans le monde où l'on est, il faut avoir eu le moyen dans l'enfance d'entrer en contact avec les origines mêmes de la civilisation dans laquelle on vit, or nous vivons dans la civilisation gréco-romaine (applaudissements) [...] La civilisation gréco-romaine est tellement la civilisation du monde dans lequel nous vivons que le Christianisme même a été la religion de l'empire romain et ce sont les siècles d'empire romain qui ont formé le christianisme $[\ldots]^{7}$.

L'importance de l'Antiquité relevait ici encore de ses liens avec le présent. Les programmes d'histoire adoptés en ig25 revinrent au parcours chronologique unique, s'étalant de nouveau sur les deux cycles de l'enseignement secondaire. L’Antiquité était alors étudiée sur deux ans, en classes de sixième et de cinquième. Un professeur de lycée, se réjouissait de ces dispositions, tout en regrettant que deux années seulement soient consacrées à l'Antiquité et en déplorant la part nouvelle accordée à l'histoire médiévale et contemporaine ${ }^{8}$. Dans la préface d'un manuel de I923, on pouvait aussi lire:

L'histoire de Rome est, dans le programme des études, la plus importante et la plus intéressante. Elle a une importance politique considérable, puisqu'il n'y a aucun fait plus grand dans les annales de l'humanité que la formation de l'empire romain, si ce n'est peut-être celle toute récente de la puissance britannique. Cette importance se double pour les nations d'Occident et pour la France en particulier du fait que notre civilisation, nos mœurs, notre caractère, notre langage, nos lois et notre droit sont d'origine romaine. Bien mieux, le sang qui coule dans nos veines n'est-il pas aussi romain que gaulois 9 ?

L'auteur réagissait lui aussi à la réduction de l'histoire romaine dans les programmes. Au même moment, d'autres soulignaient au contraire la distance entre l'époque contemporaine et l'Antiquité:

L'idéal latin n'est pas l'idéal que vous devez proposer à des hommes du xx siècle et à des citoyens de la France républicaine. Le génie latin a eu surtout pour idéal la domination de l'État romain, permettant au citoyen romain de vivre selon son goût du labeur des nations soumises; pour le citoyen de Rome, le travail était servile. Seule l'action qui conquiert, administre et exploite, était digne des Romains ${ }^{10}$.

7 Bracke, (pseudonyme d'Alexandre Marie des Rousseaux), cité par BAstiER, «La querelle des humanités...», p.5o2.

8 H. Brelet, «Le plan d'études de l'enseignement secondaire classique et les horaires», Revue internationale de l'enseignement, 77, I923, p. 85.

9 A. Piolet et J. Bernard, Histoire romaine. Classe de 5e, Paris, Vitte, ig23, introduction, p.7.

10 Intervention de Georges Leygues, le 12 mai I923, à la Chambre des députés, cité par J. BAstier, “La querelle des humanités... », p.5og. 
La différence essentielle avec le passé étant que la société romaine était basée sur l'esclavage, principe incompatible avec la République qui l'a aboli, en mai I848. Ces questions laissèrent ensuite la place à d'autres préoccupations, comme le surmenage scolaire. Les intitulés des programmes furent donc simplifiés, en Ig3I. Cette modification fit une nouvelle fois l'objet d'adaptations, comme celles proposées par Jules Isaac, alors inspecteur général:

Tout en tenant compte, autant que possible, du libellé de programmes, nous n'avons pas cru devoir sacrifier tout ce qu'ils ont éliminé: par exemple La religion romaine, la famille romaine, la Gaule avant la conquête romaine, etc. À nos collègues de décider si ces questions méritent ou non d'être étudiées ${ }^{11}$.

D'autres auteurs de manuels revendiquèrent aussi leur liberté par rapport aux programmes, allant jusqu'à remettre en cause les choix ministériels.

Il nous est même arrivé de combler les lacunes d'un programme qui, voulant être bref, en vient à oublier un des traits caractéristique de la vie des Romains. C'est ainsi que nous avons consacré un paragraphe entier au droit romain, la création la plus durable du génie des Latins. Nous estimons en effet que des enfants eux-mêmes ne doivent pas ignorer l'aspect particulier d'une culture à laquelle nous devons tant et dont nous sommes les héritiers ${ }^{12}$.

En avril Ig38, Jean Zay, alors ministre du Front populaire, reprit le système de I9o2, avec deux parcours chronologiques, un par cycle, limitant l'étude de l'Antiquité à la classe de sixième pour le collège. À la même époque, Marc Bloch, dans L'étrange défaite, ouvrage dans lequel il imputait en partie la défaite de I940 au système d'enseignement, affirmait son attachement à la culture humaniste.

Gardons-nous de réduire l'histoire, comme on a eu tendance à le faire ces dernières années, aux événements purement politiques d'une Europe, dans le temps, toute proche de nous. Le passé ancien inspire le sens et le respect des différences entre les hommes, en même temps qu'il affine la sensibilité à la poésie des destinées humaines.

L'historien prenait ainsi part au débat initié dans les années I920, sur la place accordée aux Humanités classiques, mais ici avec les arguments des Annales.

Les nombreux changements de programmes de I902, I925, i93i et ig38 pourraient donner à penser que l'on assiste à de profondes transformations, mais les contenus ne sont au fond guère modifiés, alors que l'historiographie connaît un

1 A. Alba, J. Isaac et A. Malet, Histoire romaine, à l'usage de la classe de 5e, Paris, Librairie Hachette, I93i, $7^{\mathrm{e}}$ édition.

12 А. Амввоsi, Histoire romaine pour la classe de 5e, Paris, Hatier, I929. 
important renouvellement. Les vrais changements n'intervinrent qu'après la Seconde Guerre mondiale.

Sous le régime de Vichy, en I94I, Jérôme Carcopino proposa un programme renouant avec celui de i925. Il revenait sur le parcours chronologique unique, sans céder aux partisans de la primauté de l'histoire ancienne, en dépit de son domaine de spécialité. Rome n'était alors étudiée qu'en classe de cinquième. En I944, le gouvernement provisoire ne rétablit pas les programmes de ig38, mais bien ceux de I93I. Après I945, les débats, moins nombreux, portèrent davantage sur des questions de méthode. La modification des programmes, entamée en ig57 sous l'influence de Fernand Braudel et de l'école des Annales fut immédiatement contestée. De plus, l'hostilité des associations de professeurs aboutit à un certain immobilisme, alors que les programmes définis pour une minorité de privilégiés auraient dû s'adapter à l'explosion scolaire. D'autres programmes furent donc publiés en ig6o, puis en ig63. L’histoire ancienne fut cependant peu concernée, les intitulés et les volumes horaires restant presque inchangés. Dans un livre du professeur de ig64, sous le titre «Si Rome n’avait pas existé...», les auteurs d'un manuel scolaire réaffirmaient cependant l'importance de l'étude de l'histoire romaine pour la compréhension de la langue française:

En guise d'introduction il peut être utile de montrer aux élèves que cette prodigieuse histoire d'un obscur village de paysans devenu la capitale d'un vaste empire n'est pas une histoire totalement morte, que nous en sommes encore largement tributaires. Le français vient du latin. L'histoire romaine était si familière aux hommes de la révolution française de 1789 qu'ils empruntèrent beaucoup de noms au vocabulaire politique romain ${ }^{13}$.

La filiation avec le monde romain restait l'argument principal pour justifier le maintien de la place de l'histoire romaine. L'histoire de l'Orient et de la Grèce connaissaient au même moment de semblables diminutions.

Une innovation, apparue avec le programme de I969, fut immédiatement traduite dans les manuels, comme celui qui fut édité par Masson ${ }^{14}$. Il proposait d'aborder certains thèmes de façon diachronique à l'échelle de l'Antiquité: le travail et les techniques, l'esclavage, les rapports entre art et religion, en s'appuyant sur des documents sources tant égyptiens que grecs et romains. Pour la première fois, signe de l'influence des recherches universitaires, les professeurs pouvaient déroger au continuum chronologique en envisageant les phénomènes

13 A. Alba, Rome et le Moyen Âge jusqu'en I328. 5e, Livret complémentaire, Collection Isaac, Paris, Hachette, I964.

14 R. Debon, Y. Perez, J. Portes et A. Reynaud, Histoire. L'Antiquité. Classe de 6e, Paris, Masson, I970. 
historiques à partir de l'étude des structures et de la longue durée. Cependant, cette conception de l'enseignement de l'histoire ne fit pas l'unanimité; ainsi, un inspecteur général, soulignant l'importance de l'événement et de la chronologie, exposait ses réticences face à cette approche des civilisations.

Comment peut-on dérouler dans le temps l'histoire du costume ou des moyens de communication, si la fresque des principaux événements de l'histoire n'a pas été au préalable fixée dans l'esprit des élèves? Je ne vois guère comment parler du costume grec ou romain, sans avoir jamais au préalable parlé de l'histoire grecque ou romaine. On risque d'aboutir à un enseignement qui ne repose sur rien de consistant, qui demeure pour le jeune une série d'abstractions, de figurines de musée. C'est avec Cicéron, avec Scipion l'Africain que le jeune désire entrer dans le détail de la vie romaine. Sans le récit des événements, on sort du cadre temporel, de l'enchaînement chronologique de l'histoire ${ }^{15}$.

Les témoignages montrent aussi que l'introduction de nouvelles démarches posa problème aux professeurs qui n'avaient pas été formés lors de leurs études à ces approches. La notion de civilisation devint cependant centrale dans les années 1970, les idées de Braudel ayant mis un certain temps avant d'imprégner les programmes. En ce qui concerne l'Antiquité, les programmes étaient en tout cas depuis longtemps centrés sur le bassin méditerranéen. Les débats sur la place de la chronologie, de l'événement face à l'approche civilisationnelle atteignirent leur paroxysme au moment de l'arrivée au ministère de René Haby, en mai 1974. Le ministre s'appuya sur un petit groupe d'experts, chercheurs en pédagogie et proches de l'Inspection générale, qui souhaitaient un renouvellement pédagogique et regrettaient que l'histoire soit trop axée sur la chronologie. Pour la classe où l'histoire antique est étudiée, les changements furent considérables. Les auteurs de ces programmes s'en sont expliqués a posteriori:

$[\ldots]$ en $6^{e}$, nous avions réalisé deux thèmes diachroniques en longue durée, les débuts de l'histoire jusqu'à nos jours; on voulait que les élèves qui n'ont pas en $6^{e}$ une véritable structuration du temps dans l'esprit, soient aidés à le structurer par le balayage du temps autour d'un fil directeur qui ne peut pas prendre en compte toute l'histoire mais qui est intégrateur. [...] Nous pensions qu'en ayant fait « agriculture et paysans » et "technique et communication », nous les avions magnifiquement armés en $6^{\mathrm{e}}$ sur la grande majorité des réalités de l'humanité jusqu'à la révolution industrielle ${ }^{16}$.

15 L. François, “Permanences et contraintes nouvelles», Cahiers pédagogiques, 65, I967, p. $29-32$.

16 Cité par P. Legris, “"On n’apprend plus l’histoire à nos enfants!" Retour sur la polémique de l'enseignement de l'histoire en France au tournant des années 1970-I980", in J. Barroche, N. Le BouËDec et X. Pons, Figures de l'État éducateur. Pour une approche pluridisciplinaire, Paris, L'Harmattan, 2008, p. 209. 
Les programmes étant le résultat de compromis, la chronologie conserva sa place dans les textes de I977. Pour la première fois, l'étude de la République romaine au collège fut abandonnée et l'on n'aborda plus que l'Empire romain au $\mathrm{II}^{\mathrm{e}}$ siècle. La contestation s'élargit aux universitaires, aux hommes politiques et aux médias. Le ministre avait aussi sous-estimé l'attachement des enseignants aux anciens programmes, les pesanteurs, les habitudes, et le fait que ces programmes avaient été élaborés sans les principaux intéressés.

Depuis 1977, les programmes ont été modifiés trois fois: en I985, I995 et 2008. Mais les renouvellements suggérés dans les années I970 n’ont pas été intégrés. L'histoire culturelle a cependant pris davantage de place, notamment par le biais des questions patrimoniales et, depuis 2008, avec l'intégration de l'histoire des arts à hauteur d'un quart du temps imparti à l'enseignement de l'histoire. Concernant l'Antiquité, les modifications sont minimes et chaque période conserve une place relativement équitable. L'Orient, la Grèce et Rome connaissent la même diminution horaire dans les programmes. Mais l'Égypte et Athènes sont incontestablement plus appréciées que Rome, comme l'atteste leur traitement dans les cahiers d'élèves ${ }^{17}$. Ces préférences apparaissent dès 1967 , dans les propositions d'un enseignant pour résoudre le problème de la lourdeur des programmes:

Sans renoncer à la continuité historique, ne serait-il pas plus raisonnable de passer sous silence de vastes périodes, comme l'Orient hellénistique ou le bas empire romain, dont la compréhension exige une érudition et une maturité que l'on ne peut guère rencontrer qu'à la faculté. Cela permettrait de concentrer l'attention des enfants sur les questions les plus intéressantes. Ne pourrait-on envisager un programme pour la classe de sixième comprenant deux parties: l'une obligatoire et l'autre à option? La première serait consacrée aux problèmes les plus indispensables à la culture des élèves: l'Égypte antique et Athènes au v siècle avant Jésus-Christ par exemple. La seconde fournirait un choix de sujets aux professeurs qui seraient obligés d'en traiter un ou deux. [...]. De même, en classe de Cinquième, l'accent pourrait être mis sur Rome au i ${ }^{\text {er }}$ siècle avant Jésus-Christ ${ }^{18} \ldots$

Ces mesures résonnent avec les programmes actuellement en vigueur, qui offrent la possibilité aux enseignants de faire des choix, ce qui constitue l'un des points contesté de la réforme. Ainsi, le professeur a la possibilité de traiter certaines questions optionnelles, comme Alexandre le Grand ou les savants grecs

17 A. Rodes, «Un siècle de leçons sur la conquête des Gaules à partir des cahiers d'histoire en France », in Le cartable de Clio, Revue suisse sur les didactiques de l'histoire, I3, 20I3, p. $145-154$.

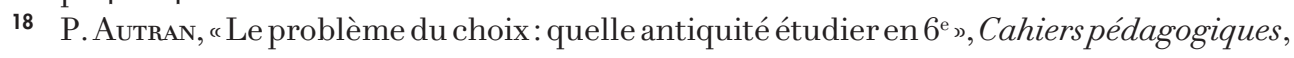
$65, \mathrm{I} 967$, p. 54 . 
au Iv siècle avant J.-C. ${ }^{19}$. En revanche, l'Égypte ne revêt plus aujourd'hui l'importance que lui concédait ce commentateur.

Ce panorama historique montre combien les récentes propositions se situent dans la continuité des changements opérés depuis le xix ${ }^{\mathrm{e}}$, quant à l'importance de la chronologie et la répartition des périodes en fonction des cycles.

\section{L'enseignement de l'histoire ancienne dans les réformes du début du $x \mathrm{XI}^{\mathrm{e}}$ siècle}

Il convient d'envisager maintenant quelles perspectives apportent les nouveaux programmes concernant l'histoire de l'Antiquité. Les réformes sont de deux ordres: l'une porte sur les cycles d'enseignement, l'autre sur les programmes.

\section{La place de l'Antiquité dans la réforme des cycles}

Les débats les plus anciens portaient sur le fait que l'enseignement de l'histoire était assuré en sixième par des professeurs de grammaire, à qui l'on reprochait, dans les années I88o, de ne pas être des spécialistes, pouvant même «inculquer des notions fausses à leurs élèves, ce qui conduit à la ruine de l'histoire ancienne ${ }^{20}$ ». Le statut des professeurs fut ensuite modifié et l'enseignement de l'histoire confié à un professeur spécifique, afin de dispenser un enseignement plus scientifique, tout en tenant compte d'une approche plus didactique.

À compter de la rentrée de 20I6, la première année de collège, la classe de sixième, est intégrée au cycle $3^{21}$, un cycle entamé à l'école élémentaire. Cette nouvelle organisation aurait pu être l'occasion de repenser le contenu des enseignements en proposant une approche chronologique nouvelle et permis de tenir compte d'une difficulté unanimement soulevée dans les revues pédagogiques et les préfaces des manuels: la maturité nécessaire pour aborder l’Antiquité. Ce point de vue était déjà mis en avant en I89o:

Quand un élève de $\mathrm{I} 3$ ans aurait compris la différence qui sépare les citoyens de l'empire, tous égaux en droits et en devoirs, de cette poignée de conquérants et d'oppresseurs qui s'appelaient sous la République les citoyens romains, n'aurait-il pas reçu la meilleure leçon d'institutions que l'on puisse comprendre à son âge ${ }^{22}$.

19 Bulletin officiel spécial, nº du 28 août 2008, p. 7 .

20 Cité par P. Marchand, L'histoire et la géographie dans l'enseignement secondaire: textes officiels, t. I. I795-I9I4, Paris, INRP, 2000, p. 59.

21 Décret $\mathrm{n}^{\circ}$ 2013-682, publié au Journal officiel du 28-7-2013.

22 H. Salomon, “L'enseignement de l'histoire dans les lycées », Revue internationale de l'enseignement, 20, I89o, p.47I. 
L'âge des élèves était encore invoqué en I970:

[...] Avoir supprimé l'étude de notions classiques évidentes pour le spécialiste surprendra plus d'un professeur. Mais ne faut-il pas tenir compte que notre enseignement s'adresse à des enfants d'une dizaine d'années ${ }^{23}$ ?

À ces difficultés s'ajoutent que les périodes abordées en classe de sixième correspondent aux temps les plus reculés: l'Orient ancien et l'Antiquité et, à compter de septembre 20I6, les débuts de l'humanité et la révolution néolithique. Ces temporalités sont difficiles à saisir par les élèves pour deux raisons essentielles. D’une part, elles se situent sur une échelle de temps très longue, le millénaire, qui plus est avant J.-C. D'autre part, parce que les notions abordées, comme l'esclavage, les colonies, la villa, n'ont pas le sens que ces mots ont aujourd'hui. Ils nécessitent donc d'être expliqués avec soin, si l'on ne veut pas que les élèves transposent dans le passé antique des conceptions contemporaines.

Les nouveaux programmes n'ont pas suffisamment pris en compte ces contraintes. Un déroulement chronologique inverse à celui actuellement institué aurait été plus pertinent. Le cycle 3 aurait ainsi pu commencer avec l'étude des périodes plus proches du présent en см1, et se terminer par l'Antiquité en sixième. Cela aurait nécessité de revenir sur une question qui n'a jamais été d'actualité: remettre en cause l'ordre chronologique établi. La première année de collège, celle où est enseignée l'Antiquité, retrouve en quelque sorte la place qu'on lui assignait au $\mathrm{xIx}^{\mathrm{e}}$ siècle: une année de transition entre l'enseignement élémentaire et l'enseignement secondaire. Le contexte est cependant bien différent. Autrefois, cette année correspondait pour de nombreux enfants à la dernière année de scolarisation, alors qu'aujourd'hui, il s'agit de mieux assurer la transition entre les deux degrés d'enseignement, à des enfants qui poursuivent presque tous leur scolarité jusqu'à ı 6 ans.

La cohérence du cycle 3 ne repose pas vraiment sur la continuité chronologique puisque les élèves abordent à deux reprises l'Antiquité en trois ans, avec une coupure d'un an pour étudier les périodes modernes et contemporaines. On pourrait se réjouir de l'importance ainsi accordée à l'Antiquité. Pourtant, étant donné l'âge des enfants (entre 8 et II ans), elle est envisagée de façon assez simple à partir d'éléments très concrets ou au contraire en s'appuyant sur des aspects mythologiques. L'histoire de l'Antiquité n'est plus revue ensuite, exceptées quelques heures pour ceux qui poursuivent en seconde générale au lycée. La logique retenue dans les nouveaux programmes consiste à envisager à deux reprises le même parcours chronologique, dans une perspective d'histoire locale et nationale à

23 R. Debon, Y. Perez, J. Portes et A. Reynaud, «Pour une refonte totale de l'histoire et de la géographie dans le secondaire », Cahiers pédagogiques, 79, décembre ı968, p.40. 
l'école élémentaire, puis dans une approche plus globale au collège. Cette distinction a suscité quelques réactions, dont le collectif Aggiornamento ${ }^{24}$ s'est fait l'écho. Si les nouveaux programmes ne tiennent pas compte de la cohérence chronologique, ils prennent, en revanche, davantage en considération la progression des apprentissages au sein du cycle, en indiquant les différences entre la classe de см1 et celle de sixième.

Les démarches initiées dès le cm1 sont réinvesties et enrichies: à partir de quelles sources se construit un récit de l'histoire des temps anciens? Comment confronter traces archéologiques et sources écrites ${ }^{25}$ ?

L'intérêt porté aux sources, en particulier archéologiques, est une opportunité pour renouveler l'enseignement de l'Antiquité et permettre aux enseignants de faire réfléchir les élèves à l'élaboration des savoirs scolaires. Mais ce n'est pas le seul atout de ces programmes.

\section{Une approche nouvelle de l'Antiquité dans les programmes de 2016}

Les programmes, qui entreront en vigueur en septembre 2or6, présentent une série de nouveautés tant dans la forme que dans les contenus. On les synthétisera dans un tableau.

Les nouveaux programmes, tout d'abord, proposent des intitulés plus vastes, avec moins de dates et de noms de personnages que ceux de 2008 (cela n'apparaît pas dans le tableau, où ne sont indiqués que les intitulés dans un souci de clarté). Il revient en général aux auteurs des manuels et des accompagnements aux programmes publiés sur le site Educsol de “traduire» les textes officiels. Dans les préfaces des manuels, les auteurs et les éditeurs avouent qu'à l'occasion des changements de textes officiels, ils privilégient les démarches les plus proches des anciens programmes pour plaire aux enseignants qui choisissent les manuels. On peut donc craindre que la mince liberté laissée par les nouveaux intitulés n'incite les enseignants à reproduire les anciennes démarches. De plus, les professeurs devront mettre en œuvre les nouveaux programmes des quatre niveaux de collège la même année, ce qui va leur demander un travail énorme et certainement les amener à réutiliser des séquences. En outre, ils devront en même temps intégrer le nouveau «socle commun de compétences, de connaissances et de culture» et le

24 De Cock Laurence, “L'Aggiornamento” et les nouveaux programmes: bilan critique et perspectives ", Cahiers d'histoire. Revue d'histoire critique [En ligne], I22, 2014, mis en

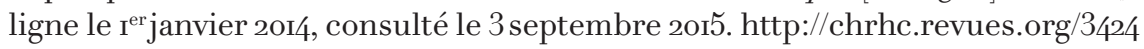

25 http://cache.media.education.gouv.fr//file/MEN_SPE_II/35/I/BO_SPE_II_26-II20I5_5o435ı.pdf, p. I74. 
nouveau programme d'histoire des arts. Enfin, le nouveau programme ne comporte pas d'indications horaires, ce qui laisse la liberté d'aborder de façon plus ou moins approfondie chacun des trois thèmes proposés. Jusqu'à présent le découpage était très strict et souvent contraignant, garantissant une égalité de traitement pour chaque période. Cependant, l'étude des cahiers d'élèves a montré la distance entre les programmes officiels et ce qui est réellement étudié en classe.

Intitulés des programmes portant sur l'Antiquité en 2008 [sans le détail des compétences] et ceux de 2or6, pour le cycle3 [cases en grisé].)

\begin{tabular}{|c|c|c|}
\hline \multirow[b]{2}{*}{ CE2 } & Programmes de $2008^{*}$ & \multirow[t]{2}{*}{ Programmes de $2016^{* *}$} \\
\hline & $\begin{array}{l}\text { Les Gaulois } \\
\text { La romanisation de la Gaule } \\
\text { La christianisation du monde } \\
\text { gallo-romain }\end{array}$ & \\
\hline CM1 & & $\begin{array}{l}\text { Thème r: Celtes, Gaulois, Grecs et Romains: } \\
\text { quels héritages des mondes anciens? }\end{array}$ \\
\hline CM2 & & \\
\hline $6^{\mathrm{e}}$ & $\begin{array}{l}\text { I. L'Orient ancien } \\
\text { 2. La civilisation grecque } \\
\text { Thème I: Au fondement } \\
\text { de la Grèce: cités, mythes, } \\
\text { panhellénisme } \\
\text { Thème } 2 \text { : La cité des Athéniens } \\
\text { ( } \mathrm{v}^{\mathrm{e}} \text {-IV's siècle): citoyenneté et } \\
\text { démocratie } \\
\text { Thème } 3 \text { : Alexandre le Grand ou } \\
\text { la Grèce des savants } \\
\text { 3. Rome } \\
\text { Thème I: Des origines à la fin } \\
\text { de la République: fondation, } \\
\text { organisation politique, conquêtes } \\
\text { Thème } 2 \text { : L'Empire: l'empereur, } \\
\text { la ville, la romanisation } \\
\text { 4. Les débuts du judaïsme et du } \\
\text { christianisme }\end{array}$ & $\begin{array}{l}\text { Thème I: La longue histoire de l'humanité } \\
\text { et des migrations } \\
\text { - Premiers États, premières écritures. } \\
\text { Thème } 2 \text { : Récits fondateurs, croyances et } \\
\text { citoyenneté dans la Méditerranée antique } \\
\text { au I'r millénaire avant J.-C. } \\
\text { - Le monde des cités grecques } \\
\text { - Rome du mythe à l'histoire } \\
\text { - La naissance du monothéisme juif dans un } \\
\text { monde polythéiste } \\
\text { Thème 3: L'Empire romain dans le monde } \\
\text { antique } \\
\text { - Conquêtes, paix romaine et romanisation } \\
\text { - Des chrétiens dans l'Empire } \\
\text { - Les relations de l'empire romain avec les } \\
\text { autres mondes anciens: l'ancienne route de } \\
\text { la soie et la Chine des Han }\end{array}$ \\
\hline
\end{tabular}

* http://www.education.gouv.fr/bo/20o8/hs3/programme_CE2_CMI_CM2.htm - pour l'école élémentaire et http://media.education.gouv.fr/file/special_6/5ı/6/Programme_hist_geo_education_civique_6eme_335ı6.pdf pour le collège.

** http://cache.media.education.gouv.fr//file/MEN_SPE_II/35/I/BO_SPE_II_26-II-20I5_50435I.pdf p.I78.

Le nouveau programme présente aussi une approche très nouvelle pour l'étude de l'Antiquité au collège, selon des modalités qui n'avaient jamais encore été envisagées. La principale nouveauté concerne le découpage des différentes périodes 
antiques, qui avaient jusqu'alors toujours été proposées comme des entités distinctes. Elles sont désormais associées dans des thématiques synchroniques. Ainsi, dans le thème 2, “Récits fondateurs, croyances et citoyenneté dans la Méditerranée antique au Ir millénaire avant J.-C.», il est proposé d'étudier les cités grecques, la fondation de Rome et les débuts du judaïsme. L'objectif est de montrer la concomitance chronologique de ces trois «civilisations». Pour la première fois, les mondes grec et romain sont associés. De même, le thème3, «L'Empire romain dans le monde antique », permet de mettre en relation des leçons qui étaient habituellement distinctes, comme la romanisation et les débuts du christianisme. Le texte du programme insiste sur

des représentations globales des mondes explorés. [...] Les professeurs s'attachent à montrer les dimensions synchronique ou diachronique des faits étudiés. Les élèves poursuivent ainsi la construction de leur perception de la longue durée ${ }^{26}$.

Cette approche, très critiquée en I977, n’a pas, à ma connaissance, été contestée récemment, sans doute, parce que cette vision du monde antique fait écho aux travaux de Paul Veyne sur L'empire gréco-romain ${ }^{27}$, mais aussi parce qu'elle est davantage synchronique que diachronique. En ce qui concerne plus précisément le monde romain, le découpage traditionnel entre la République et l'Empire n'apparaît plus. La République disparaît des intitulés et n'est abordée qu'à l'occasion de la légende de fondation de Rome ou lors des conquêtes. La période de la République avait déjà disparu des programmes de 1977, avant d'être immédiatement restaurée.

Un second changement mérite attention. Le judaïsme et le christianisme ne sont plus présentés exclusivement comme les premiers monothéismes, mais sont inscrits dans deux thèmes antiques plus larges. Le contexte historique prévaut donc sur le fait religieux, ce qui est souligné dans le préambule des programmes: “L'étude des faits religieux ancre systématiquement ces faits dans leurs contextes culturel et géopolitique ${ }^{28}$. » La modification des intitulés, entre le mois d'avril et le mois de septembre, le confirme. Les chapitres initialement intitulés «Les débuts du judaïsme » et «Les débuts du christianisme» sont désormais libellés «La naissance du monothéisme juif dans un monde polythéiste» et “Des chrétiens dans l'Empire », ce qui modifie considérablement la façon d'envisager ces questions.

Globalement, dans le programme d'histoire, la période romaine est peu modifiée dans ses contenus, et c'est davantage le découpage chronologique qui est concerné, alors que d'autres périodes antiques connaissent des modifications plus

26 Programme 20I6, op. cit., p. I74.

27 P. Veyne, L'Empire gréco-romain, Paris, 2012.

28 Programmes de 20I6, p. I74. 
profondes. La préhistoire, qui avait disparu des programmes depuis 1995, retrouve une place timide, justifiée par ses liens avec les disciplines scientifiques. Quant à l'absence de la protohistoire, notons une fois encore, à la suite de Christian Goudineau, que «l'on ne retrouvera la Gaule que lorsque César aura la bonté de la conquérir ${ }^{29}$ ». L'histoire gauloise n'est donc toujours pas abordée au collège en tant que telle dans les programmes officiels. Au-delà du cM1, elle n'est envisagée que dans le cadre des conquêtes romaines.

La Grèce et l'Égypte pâtissent davantage du nouveau programme. Même si la Grèce peut désormais être rapidement abordée à l'école élémentaire, la place qui lui est consacrée au collège est considérablement réduite et limitée à l'étude de la cité. L'Égypte conserve la place dévolue dans les programmes de 2008: une civilisation moins intemporelle, mais liée à deux faits majeurs, l'apparition des premiers états et celle des premières écritures, faits que l'enseignant peut aussi choisir d'envisager à partir d'une cité mésopotamienne. L'Égypte était susceptible de trouver sa place dans l'étude de la période hellénistique, mais le cours optionnel sur Alexandre le Grand ou les savants du rve siècle avant J.-C. a disparu. Ce choix est regrettable dans la mesure où cette leçon était l'une des rares occasions d'envisager les liens anciens entre l'Occident et l'Orient et de rendre plus intelligible le présent.

L'ouverture vers des horizons lointains est maintenue avec l'étude de la Chine des Han, qui est mieux reliée à l'ensemble du programme qu'en 2008, en mettant en avant ses liens avec l'Empire romain. Ce rapprochement renoue cependant avec une vision européo-centrée de l'étude de l'Antiquité, qui a souvent été dénoncée.

\section{Conclusion}

Les nouveaux programmes d'histoire suscitent aujourd'hui encore de vives réactions. Certains arguments perdurent depuis la fin du xix siècle, même si, sur le long terme, le désintérêt pour l'histoire antique est flagrant. Les programmes qui devront être appliqués en septembre 2016 ont été peu commentés pour l'Antiquité et le projet remis en avril 2015 n'a quasiment pas été modifié pour cette période. Ce manque d'intérêt n'est pas étranger à la place aujourd'hui faite aux lettres classiques; leur mise en péril a néanmoins provoqué davantage de protestations. Pourtant, l'intégration au cycle 3 de la classe dans laquelle est enseignée l'Antiquité, la classe de sixième, n'a pas donné lieu au renouvellement des contenus qui aurait été nécessaire. L'histoire romaine est abordée à deux reprises en deux ans, sans réelle cohérence. Il faut en revanche noter combien ces nouveaux programmes d'histoire marquent une rupture importante avec tous les programmes antérieurs.

29 C. Goudineau, César et la Gaule, Errance, Paris, I99o, p. I9-20. 
Pour la première fois, l'approche chronologique l'emporte sur les espaces géographiques et les civilisations, selon lesquels est habituellement découpée l'Antiquité: les cités grecques sont abordées dans le même thème que l'Urbs, les débuts du judaïsme et du christianisme sont étudiés dans leur contexte historique avant d'être considérés pour leurs aspects religieux. Ces changements, passés inaperçus, traduisent pourtant une approche nouvelle de l'histoire antique dans le cadre scolaire, envisagée comme un tout et moins comme une période cloisonnée en domaines spécialisés. Enfin, on peut se réjouir que l'étude des sources et leur confrontation soit soulignée et espérer qu'elle débouche sur des démarches laissant plus de place à l'historiographie.

\section{Aurélie RoDEs}

Professeure d'histoire géographie

Docteure en histoire

UT2J - PLH-ERASME

aurelie.rodes@univ-tlse2.fr 